\title{
Multiple-scattering approach to finite-sized photonic band-gap materials
}

\author{
Lie-Ming $\mathrm{Li}^{*}$ and Zhao-Qing Zhang \\ Department of Physics, Hong Kong University of Science and Technology, Clear Water Bay, Kowloon, Hong Kong, China
}

(Received 4 May 1998)

\begin{abstract}
Multiple-scattering theory is used to study the transmission, scattering, and radiation properties of finitesized photonic band-gap materials in two dimensions. By adopting a source with a finite beam width to simulate realistic experimental conditions, we are able to obtain transmission results that are in excellent quantitative agreements with the measured data. Also, we find that the apparent gaps seen by an antenna can be much larger than the full gaps of the system. Inside an apparent gap, analytic results have been obtained for the radiation patterns and the total radiation power. [S0163-1829(98)00139-8]
\end{abstract}

Materials with a photonic band gap (PBG) have been under intensive studies both theoretically and experimentally over the past few years. ${ }^{1-9}$ The existence of gaps, which prohibit the propagation of electromagnetic waves, can have important impacts both in science and technology. Among other important issues, the study of transmittance, the most measured quantity, and the radiation patterns of an antenna on a PBG substrate are also the foci of much studies. In these studies, the finite-difference method is commonly used., ${ }^{1,4}$ However, this method requires large storage and heavy CPU time. Also, various boundary conditions have to be imposed by hand. Very recently, the multiple-scattering theory has been used to study the transmission and defect properties of finite PBG samples. ${ }^{9}$ The multiple-scattering method treats finite PBG samples as scattering objects in open geometry. The radiation boundary condition is naturally imposed. We have independently adopted this method to study the transmission, scattering, and radiation properties of finite PBG samples. In the case of transmission, we used a source with a finite beam width to simulate the realistic experimental situations. In this case, a generalized transmission coefficient can be defined in terms of the far-field total scattering amplitude. We demonstrate explicitly that this method can produce transmission results that are in excellent quantitative agreements with the available experimental data for both $S$ and $P$ waves. From the total scattering amplitude we can retrieve the dispersion relations and the decay length inside a gap. The dependence of total scattering amplitude on the beam width is also discussed. Our calculation of transmission coefficient is different from that of Ref. 9 where plane waves were used as the incident beam and the near-field flux was used to calculate the transmittance. In the case of radiation, we find that the apparent gaps seen by an antenna can be much larger than the full gaps of the system. Inside an apparent gap, analytic expressions have been obtained for the radiation pattern and total radiation power.

Below we give a brief derivation of the multiplescattering method in a form that applies to both $S$ and $P$ waves. Consider a system of $N$ identical cylinders of radius $R$ and dielectric constant $\epsilon$ under an external source $u_{\text {inc }}(\vec{\rho})$ at a fixed frequency $\omega=2 \pi f$. Here $\vec{\rho} \equiv(\rho, \theta)$ specifies the position in a two-dimensional plane. In the case of TM (TE) mode, $u$ denotes the electric (magnetic) field. Let us first consider the scattering of a particular cylinder $j$ located at $\vec{\rho}_{j}=\left(\rho_{j}, \theta_{j}\right)$. The incident waves come from both the external source $u_{\text {inc }}$ and the radiation of other cylinders. The total field outside the cylinder $j$ can be written as the sum of the incident and scattered fields, i.e.,

$$
u(\vec{\rho})=\sum_{m=-M}^{M}\left[\alpha_{m}(j) J_{m}\left(k_{0} \rho_{\rho j}\right)+\widetilde{B}_{m}(j) H_{m}\left(k_{0} \rho_{\rho j}\right)\right] e^{i m \theta_{\rho j}}
$$

where $k_{0}=\omega / c, \vec{\rho}_{\rho j}=\vec{\rho}-\vec{\rho}_{j}=\left(\rho_{\rho j}, \theta_{\rho j}\right), M=\infty$, and $J_{m}$ and $H_{m}$ are, respectively, the Bessel function and the Hankel function of the first kind. The ratio $D_{m}(j)=\widetilde{B}_{m}(j) / \alpha_{m}(j)$ has the form ${ }^{10}$

$$
D_{m}(j)=\frac{k J_{m}\left(k_{0} R\right) J_{m}^{\prime}(k R)-k_{0} J_{m}^{\prime}\left(k_{0} R\right) J_{m}(k R)}{k_{0} J_{m}(k R) H_{m}^{\prime}\left(k_{0} R\right)-k H_{m}\left(k_{0} R\right) J_{m}^{\prime}(k R)}
$$

for the $S$ wave (TM mode), where $k=k_{0} \sqrt{\epsilon}$. Similar expression can be found for the $P$ wave (TE mode). If we also expand the external field from the $i$ th cylinder as

$$
u_{\mathrm{inc}}(\vec{\rho})=\sum_{m=-M}^{M}\left[\alpha_{m}^{(0)}(i) J_{m}\left(k_{0} \rho_{\rho i}\right)\right] e^{i m \theta_{\rho i}}
$$

$\alpha_{m}(i)$ can be expressed as the sum of $\alpha_{m}^{(0)}(i)$ and contributions from other cylinders that depend linearly on $\widetilde{B}_{l}(j)$. With the use of the Graf's addition theorem, ${ }^{11}$ a set of selfconsistent equations for $\widetilde{B}_{m}(i)$ can be obtained:

$$
\begin{aligned}
\widetilde{B}_{m}(i)= & \alpha_{m}^{(0)}(i) D_{m}(i) \\
& +\sum_{j \neq i} \sum_{l=-M}^{M} \widetilde{B}_{l}(j) D_{m}(i) e^{i(l-m)\left(\theta_{i j}+\pi\right)} H_{l-m}\left(k_{0} \rho_{i j}\right),
\end{aligned}
$$

where $\vec{\rho}_{i j}=\vec{\rho}_{j}-\vec{\rho}_{i}=\left(\rho_{i j}, \theta_{i j}\right)$. After solving Eq. (4), we rewrite Eq. (1) as $u=u_{\text {inc }}+u_{\text {scatt }}$, where the total scattered field is 


$$
u_{\text {scatt }}(\vec{\rho})=\sum_{j=1}^{N} \sum_{m=-M}^{M} \widetilde{B}_{m}(j) H_{m}\left(k_{0} \rho_{\rho j}\right) e^{i m \theta_{\rho j}} .
$$

The near-field radiation pattern can be obtained from the Poynting vector

$$
\vec{S}(\vec{\rho})=\frac{-c}{8 \pi k_{0}} \operatorname{Im}\left[u(\vec{\rho}) \nabla u^{*}(\vec{\rho})\right] .
$$

In the far field, when $k_{0} \rho \gg 1$, the asymptotic behavior of $u_{\text {scatt }}$ in Eq. (5) becomes $u_{\text {scatt }}(\vec{\rho}) \rightarrow f_{s}(\theta) \exp \left(i k_{0} \rho\right) / \sqrt{\rho}$, where the total scattering amplitude has the form

$$
\begin{aligned}
f_{s}(\theta)= & \sqrt{\frac{2}{\pi k_{0}}} e^{-i \pi / 4} \sum_{i=1}^{N} \sum_{m=-M}^{M} e^{-i k_{0} \rho_{i} \cos \left(\theta_{i}-\theta\right)} \\
& \times(-i)^{m} e^{i m \theta} \widetilde{B}_{m}(i) .
\end{aligned}
$$

By using sources with different profiles, we are able to study, from $f_{s}(\theta)$, the transmission and radiation properties of a PBG sample.

For the case of transmission, a source with a beam width smaller than the sample width is required to avoid diffraction. One way to prepare such a probe beam is to put an aperture in front of the sample. ${ }^{7}$ In this case the incident field can be obtained from the Kirchhoff integral formula. ${ }^{12}$ In two dimensions, for a plane wave, $\exp \left(i k_{0} x\right)$, incident from $x<0$, the diffracted wave in the region $x>0$ arising from a slit centered at the origin with an opening of width $w$ in the $y$ direction is given by

$$
u_{\text {inc }}(x, y)=\left(\frac{k_{0}}{4}\right) \int_{-w / 2}^{w / 2} d y^{\prime}\left[H_{0}\left(k_{0} \rho^{\prime}\right)+i \frac{x}{\rho^{\prime}} H_{1}\left(k_{0} \rho^{\prime}\right)\right],
$$

where $\rho^{\prime}=\sqrt{x^{2}+\left(y-y^{\prime}\right)^{2}}$. It can be shown that, when $\rho \gg w$, Eq. (8) becomes $u_{\text {inc }}(\vec{\rho}) \sim a_{0}(\theta) \exp \left(i k_{0} \rho\right) / \sqrt{\rho}$, with

$$
a_{0}(\theta)=e^{-i \pi / 4} \sqrt{\frac{k_{0}}{2 \pi}} \cos ^{2}(\theta / 2) \frac{2 \sin \left[k_{0}(w / 2) \sin \theta\right]}{k_{0} \sin \theta} .
$$

From Eq. (6), the far-field energy flux has the form

$$
\vec{S}(\rho, \theta)=\frac{c\left[\left|a_{0}(\theta)+f_{s}(\theta)\right|\right]^{2}}{8 \pi \rho} \hat{\rho}=S(\theta) \frac{\hat{\rho}}{\rho} .
$$

Here, we define a generalized transmission coefficient as the ratio of energy flux of Eq. (10) to that of the incident wave at $\theta=0$, i.e.,

$$
T=\left|1+\frac{f_{s}(0)}{a_{0}(0)}\right|^{2}=\left|1+\sqrt{\frac{2 \pi}{k_{0} w^{2}}} e^{i \pi / 4} f_{s}(0)\right|^{2} .
$$

In order to demonstrate the validity of this approach, we chose the experimental system in Ref. 3. The sample was made of 0.74-mm-diameter alumina-ceramic rods with the dielectric constant 8.9 arranged in a square array with the lattice constant $1.87 \mathrm{~mm}$. There were 7 rows of rods in the $x$ direction (propagation direction) and 25 columns in the $y$ direction. In order to make comparison with the experiment, we copy the measured amplitude spectra (in arbitrary units)

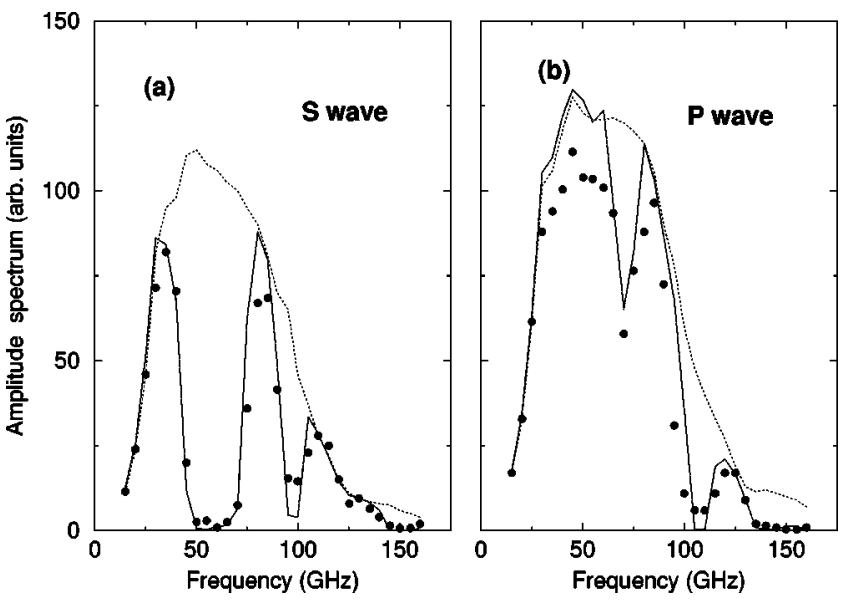

FIG. 1. The transmission amplitude for the experimental system of Ref. 3. The solid circles denote the measured data. The dashed curves are the reference transmission in the absence of the sample. The solid curves are the calculated results. (a) For $S$ wave (TM mode); (b) for $P$ wave (TE mode).

in the absence of sample by two dashed curves in Figs. 1(a) and 1(b) for the cases of $S$ and $P$ waves, respectively. The measured transmission data are denoted by solid circles. For the probe beam, we have assumed that a slit of width $w$ $=30 \mathrm{~mm}$ is placed at a distance $l=20 \mathrm{~mm}$ in front of the sample. The calculated $\sqrt{T}$ is multiplied by the reference amplitude spectra and then plotted by two solid curves in Fig. 1. For the frequency range of interest here, our calculations converge with $M=2$. For both $S$ and $P$ waves, we find excellent agreements between theory and experiments. These results are insensitive to the precise values of $w$ and $l$. For the cases with defects and randomness, we also found good agreements between theory and experiments. ${ }^{2,7}$

To discuss the scattering and radiation behaviors, we chose a sample of 5 rows (in the $x$ direction) and 25 columns arranged in a square array. Here, we consider only the case of $S$ waves. The results obtained here apply to the case of $P$ waves as well. For simplicity, we set the lattice constant $d$ as the length unit. The radius and dielectric constant of the rod are $R=0.38 d$ and $\epsilon=8.9$, respectively. These parameters are chosen to produce full gaps in the system. The band structure of a periodic system was given in Ref. 8. We have reproduced this band structure by solving the secular equation (4) with the use of the Bloch theorem. ${ }^{5,6}$ The results are plotted in Fig. 3(b), where the frequency $f$ is in units of $c / d$. By using a slit of width $w=10 d$ at a distance $l=5 d$ in front of the sample, we calculated the transmission coefficient $T(f)$ from Eq. (11). The result is plotted in curve $A$ of Fig. 2. The location of band gaps agrees well with that shown in the band structures. In curves $B$ and $C$, we plot the functions $\ln \left|f_{s}(0) / \sqrt{d}\right|$ and $\ln \left(\sigma_{t} / d\right)$. Inside the band gaps, these functions are structureless. This can be seen by taking $T \approx 0$ in Eq. (11), which gives $\left|f_{s}(0)\right| / \sqrt{d} \approx \sqrt{f}(w / d)$. Inside the bands, $\left|f_{s}(0)\right|$ shows large variations in frequency. This is related to the phase shift of the scattered waves. If we assume $T \approx 1$ in the band, from Eq. (11), we find $f_{s}(0) / a_{0}(0) \approx 2 i \sin (\phi / 2) \exp [i \phi / 2]$ and $\left|f_{s}(0)\right| / \sqrt{d}$ $\approx 2|\sin (\phi / 2)| \sqrt{f}(w / d)$, where $\phi$ is the phase difference between outgoing and incoming waves. $\phi$ changes rapidly near 


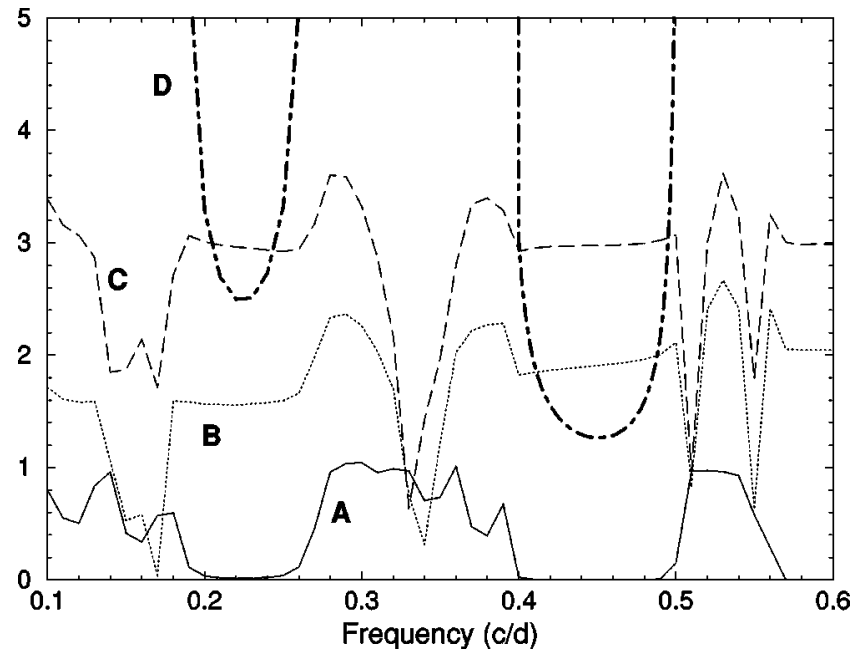

FIG. 2. The scattering properties of a photonic band gap sample described in the text under an external source with a finite beam width $w=10 d$. Curves $A, B$, and $C$ are, respectively, the transmission coefficient $T$, the logarithm of dimensionless scattering amplitude $\ln \left|f_{s}(0) / \sqrt{d}\right|$, and total scattering cross section $\sigma_{t}$. Curve $D$ denotes the decay length $\xi / d$ inside the band gaps.

band edges. The derivative of $\phi(f)$ gives information on group velocity $v_{g}$. At band edge, $d \phi / d f$ diverges and $v_{g}$ approaches zero. Thus, from $f_{s}(0)$ we are able to extract the effective dielectric constant for frequencies inside a band and the decay length for frequencies inside a gap. More precisely, we consider the PBG sample as a homogeneous medium of thickness $L$ with an effective complex index of refraction $n_{e}=n^{\prime}+i n^{\prime \prime}$. By using the standard transmission formula for a $\operatorname{slab}^{9}$ and the calculated $t=1+f_{s}(0) / a_{0}(0)$ from Eq. (11), we are able to determine $n_{e}$. Inside a band we find $n^{\prime \prime}=0$. The function $n^{\prime}(f)$ gives the dispersion relations in the $x$ direction, which is shown by heavy circles in Fig. 3(b). Inside the gaps, the waves become evanescent and

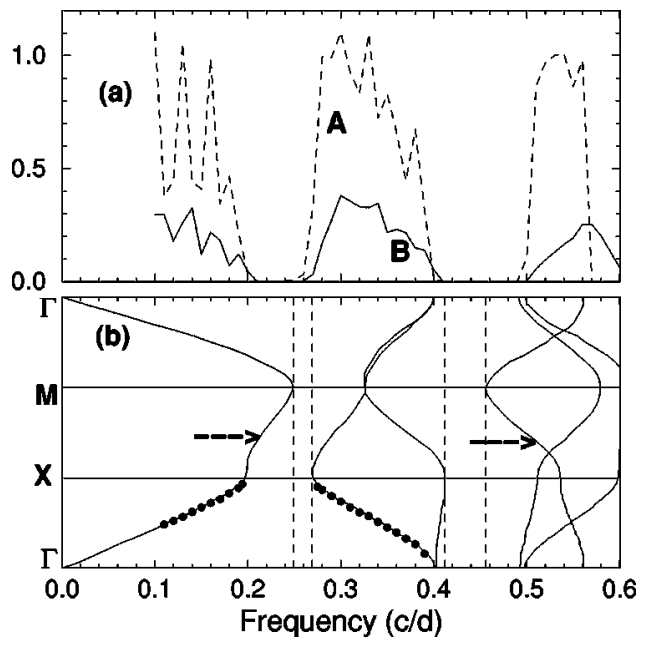

FIG. 3. For the system described in Fig. 2. (a) The scattering properties obtained from a point source incidence. Curve $A$ is the generalized transmission coefficient. Curve $B$ is the dimensionless total transmitted power. (b) The band structures reproduced from Ref. 8. The arrows indicate the positions of the apparent gaps and the solid circles are the dispersion relation obtained from transmission calculations. the decay of $|t|$ is described by a nonzero $n^{\prime \prime}$. The decay length $\xi$ can be obtained from $\xi / d=1 / 2 \pi f n^{\prime \prime}$, which is plotted by curve $D$ in Fig. 2. This result is confirmed by independent calculations of $\sqrt{T}$ as a function of $L$. In the case when reflectance $R=\left[\left(n^{\prime}-1\right) /\left(n^{\prime}+1\right)\right]^{2}$ is small, we recover the well-known relation $n^{\prime}=1+(\phi d / 2 \pi f L){ }^{3}$ The structure of $\sigma_{t}$ is similar to that of $\left|f_{s}(0)\right|$. Inside the band gaps, we find $\sigma_{t} \approx 2 w=20 d$. This confirms that the PBG sample actually acts as a perfect reflector. The magnitude of $\sigma_{t}$ increases with the beam width $w$ and saturates to the value of sample width $W$ when $w>W$.

We now consider a source close to the sample to represent an antenna. The incident field of a most general source located at origin can be expressed as

$$
u_{\mathrm{inc}}(\vec{\rho})=\sum_{m=-\infty}^{\infty} A_{m} H_{m}\left(k_{0} \rho\right) e^{i m \theta} .
$$

In the far field, we have $u_{\text {inc }}(\vec{\rho}) \sim a_{0}(\theta) \exp \left(i k_{0} \rho\right) / \sqrt{\rho}$, where

$$
a_{0}(\theta)=\sqrt{\frac{2}{\pi k_{0}}} \sum_{m=-\infty}^{\infty} A_{m} e^{-i \pi / 4}(-i)^{m} e^{i m \theta} .
$$

Here, we consider the simplest case of a point source, i.e., $A_{m}=\delta_{m, 0}$. In this case, $a_{0}=\sqrt{2 / \pi k_{0}} \exp (-i \pi / 4)$ is $\theta$ independent. A sample consisting of 7 rows and 97 columns is placed at a distance $l=5 d$ away from the point source. $l$ measures the distance from the source to the surface of the sample that passes through the center of each cylinder in the first row. For the forward scattering, we have calculated the generalized transmission coefficient, $T=\left|1+f_{s}(0) / a_{0}\right|^{2}$, and plotted in curve $A$ of Fig. 3(a). The locations of two gaps agree well with the result of a finite beam width (curve $A$ of Fig. 2) and the prediction of the band structures, i.e., (0.197, $0.269)$ and $(0.412,0.492)$. For total transmitted power, $P_{T}$ $=2 \int_{0}^{\pi / 2} S(\theta) d \theta$, it is expected that the location of gaps should be determined by the location of the full gaps of the system as waves are now incident from different angles. From the band structures, the positions of full gaps are $(0.249,0.269)$ and $(0.412,0.456)$ as indicated by the vertical dashed lines in Fig. 3(b). In curve $B$ of Fig. 3(a), we plot the dimensionless transmitted power $\bar{P}_{T}=\left(2 \pi k_{0} / c\right) P_{T}$. The positions of gaps in $\bar{P}_{T}(f)$ seem to coincide with that of $T(f)$ in curve $A$. Thus, the apparent gaps in $P_{T}$ are much larger than the full gaps of the system. The existence of these larger gaps is due to refraction. If we use the effective index of refraction $n_{e} \approx 2.4$ at the lower band edge of the $X$ point and Snell's law, the upper bound for the propagation direction in the PBG material is $\theta_{c}=\sin ^{-1}\left(1 / n_{e}\right) \approx 24.6^{\circ}$. This angle corresponds to a point between $X$ and $M$ points in the band structures as indicated by arrows in Fig. 3(b). All the modes above these arrows cannot be excited. Therefore, the apparent gaps become $(0.21,0.269)$ and $(0.412,0.492)$.

The difference between an apparent gap from $P_{T}$ and a gap from $T$ becomes more transparent from the angular distribution of flux. In Fig. 4, we plot the angular distribution of the dimensionless flux $\bar{S}(\theta)=\left(2 \pi k_{0} / c\right) S(\theta)$ at $f=0.20$, 0.23 , and 0.31 in curves $A, B$, and $C$, respectively. The case of $f=0.20$ falls in the gap of $T$, but not $P_{T}$. This is indicated by the small value of $\bar{S}$ at $\theta=0$ and a peak near $\theta=67^{\circ}$ in 


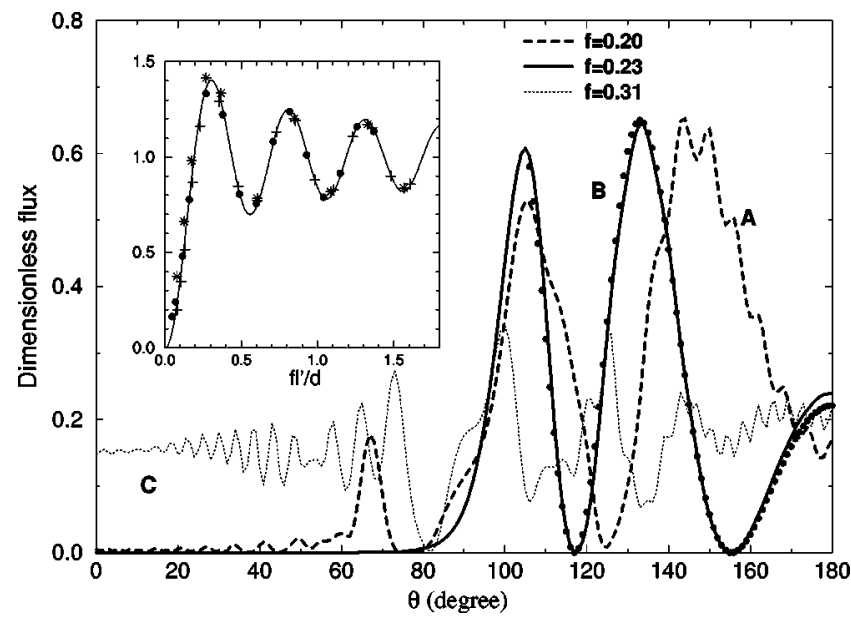

FIG. 4. For a point source incidence, the angular distribution of dimensionless flux at frequencies $f=0.20$ (curve $A$ ), 0.23 (curve $B$ ), and 0.31 (curve $C$ ). The solid circles are the analytic results. Inset: The dimensionless radiation power as a function of distance $f l^{\prime} / d$ at $f=0.22$ (circles), 0.25 (crosses), and 0.48 (stars). The solid curve represents the analytic result.

curve $A$. Inside the apparent gap of $P_{T}$, although $f=0.23$ falls outside the full gap, nevertheless, curve $B$ shows that all waves are reflected. For curve $C$, the frequency lies inside a band and the flux covers all directions. Since the PBG material acts like a perfect reflector for frequencies inside the apparent gaps, e.g., curve $B$ of Fig. 4, the angular oscillations in the reflected flux can be obtained by replacing the sample with a perfect reflector at a distance $l^{\prime}=l-\delta$ from the source. From the method of image, we find the dimensionless flux $\bar{S}_{a}(\theta)=\left[1-\cos \left(4 \pi f l^{\prime} \cos \theta / d\right)\right] / \pi$. By choosing $\delta \approx 0.22 d$, we plot $\bar{S}_{a}$ by solid circles in Fig. 4. They coincide excellently with curve $B$. This analytic result is valid as long as $l^{\prime}$ is greater than half of the wavelength $\lambda$, or $l^{\prime} / d$ $>1 / 2 f$. The value of $\delta$ is a constant for different $l$ but varies with frequency. For all the frequencies inside the two apparent gaps shown in Fig. 3, we find $0<\delta<R=0.38 d$. We have also found that the radiation pattern is insensitive to the change of source position along the $y$ axis even when $l^{\prime}$ $\approx \lambda / 2$. This may be due to the fact that the $\lambda$ studied here is larger than the length of microstructure.

In the inset of Fig. 4, we plot in solid circles the dimensionless total radiation power $\bar{P}=2 \int_{\pi / 2}^{\pi} \bar{S}(\theta) d \theta$ as a function of $f l^{\prime} / d$ at $f=0.22$ (circles), 0.25 (cross), and 0.48 (stars) inside the apparent gaps. Also plotted in the inset (solid curve) is the analytic result $\bar{P}_{a}=1-J_{0}\left(4 \pi f l^{\prime} / d\right)$. It is interesting to see that $\bar{P}$ follows the analytic results even when $f l^{\prime} / d<1 / 2$. Although when $f l^{\prime} / d<1 / 2$, the source sees the microstructure and PBG sample cannot be considered as a perfect reflector, nevertheless, the effects due to microstructure seem to average out and do not contribute to the total radiation power. It is expected that the discussions here are also valid for more complicated sources in Eq. (12).

The authors wish to thank C. T. Chan, P. Sheng, and D. Zhang for many useful discussions. This work was supported by Hong Kong RGC Grant No. HKUST 6137/97P.
*Permanent address: Department of Modern Applied Physics, Tsinghua University, Beijing 100084, China.

${ }^{1}$ For example, Photonic Band Gap Materials, edited by C. M. Soukoulis (Kluwer Academic, Dordrecht, 1996); E. Yablonovitch, Phys. Rev. Lett. 58, 2059 (1987); S. John, ibid. 58, 2486 (1987); K. M. Ho, C. T. Chan, and C. M. Soukoulis, ibid. 65, 3152 (1990).

${ }^{2}$ S. L. McCall, P. M. Platzman, R. Dalichaouch, D. Smith, and S. Schultz, Phys. Rev. Lett. 67, 2017 (1991).

${ }^{3}$ W. M. Robertson, G. Arjavalingam, R. D. Meade, K. D. Brommer, A. M. Rappe, and J. D. Joannopoulos, Phys. Rev. Lett. 68 , 2023 (1992).

${ }^{4}$ J. B. Pendry and A. MacKinnon, Phys. Rev. Lett. 69, 2772 (1992).
${ }^{5}$ K. M. Leung and Y. Qiu, Phys. Rev. B 48, 7767 (1993).

${ }^{6}$ N. A. Nicorovici, R. C. McPhedran, and L. C. Botten, Phys. Rev. E 52, 1135 (1995).

${ }^{7}$ H. Li, B. Cheng, and D. Zhang, Phys. Rev. B 56, 10734 (1997).

${ }^{8}$ J. D. Joannopoulos, R. D. Meade, and J. N. Winn, Photonic Crystals-Molding the Flow of Light (Princeton University Press, Princeton, NJ, 1995).

${ }^{9}$ G. Tayeb and D. Maystre, J. Opt. Soc. Am. A 14, 3323 (1997).

${ }^{10}$ H. C. van de Hulst, Light Scattering by Small Particles (Dover, New York, 1981).

${ }^{11}$ Handbook of Mathematical Functions, edited by M. Abramowitz and I. A. Stegun (Dover, New York, 1972), p. 363.

${ }^{12}$ For example, J. D. Jackson, Classical Electrodynamics (Wiley, New York, 1975). 